\title{
Tangence
}

\section{L'épopée d'une famille hors-la-loi}

\section{Martin Poirier}

Numéro 47, mars 1995

Écritures au féminin : le genre marqué

URI : https://id.erudit.org/iderudit/025854ar

DOI : https://doi.org/10.7202/025854ar

Aller au sommaire du numéro

Éditeur(s)

Tangence

ISSN

0226-9554 (imprimé)

1710-0305 (numérique)

Découvrir la revue

Citer cet article

Poirier, M. (1995). L'épopée d'une famille hors-la-loi. Tangence, (47), 98-111.

https://doi.org/10.7202/025854ar d'utilisation que vous pouvez consulter en ligne.

https://apropos.erudit.org/fr/usagers/politique-dutilisation/ 


\section{L'épopée d'une famille hors-la-loi}

\section{Martin Poirier, UQAM}

Le roman Des cailloux blancs pour les forêts obscures ${ }^{1}$, le dernier volet de la trilogie romanesque autobiographique de Jovette Marchessault, surprend par la multitude de genres littéraires auxquels il se rattache. En effet, il se présente d'emblée comme un roman familial, mais il intègre également l'épopée, le fantastique et l'autobiographie. De plus, par sa redéfiniton du rôle de héros et d'héroüne, comme nous le verrons, et son désir de transformer les relations entre les hommes et les femmes, il s'inscrit également dans la lignée des romans féministes et utopiques. Il remet en cause les frontières du roman traditionnel et confronte l'institution littéraire qui, selon Lucie Robert, s'inscrit "dans ces formes fixes, dans les genres eux-mêmes, en tant que modèles canoniques et en tant que normes littéraires de la vraisemblance” 2 .

La rencontre de l'écrivaine lesbienne, Jeanne, de son amante, Noria, et du père de cette dernière, le Lion de Bangor, permet qu'à tour de rôle chacun puisse se raconter. $\dot{A}$ ces personnages se joignent également la mère de Noria et son amante, Harriet. Au fil de leurs récits se dévoile l'histoire d'une famille qui prend les allures d'une grande épopée familiale transposée dans un futur idéalisé, très près de notre présent. Nous verrons comment ce roman subvertit la structure de la famille traditionnelle - voire patriarcale - tout en questionnant les structures du roman traditionnel grâce entre autres à une intégration particulière du surnaturel et de l'oralité propres à l'épopée ${ }^{3}$.

1 Jovette Marchessault, Des cailloux blancs pour les forêts obscures, Montréal, Leméac, 1987, $165 \mathrm{p}$.

2 Lucie Robert, Alphonsine Moisan ou Emma Bovary? Le choix douleureux [sic] d'Émilie Bordeleau, Claudine Potvin et Janice Williamson (dir.), Women's Writing and the Literary Institution/L'écriture au féminin et l'institution littéraire, Alberta; University of Alberta, 1992, p. 161.

3 Cette approche s'inspire du travail de Marianne Hirsch qui propose une relecture féministe de la notion de - roman familial. de Freud afin d'étudier les liens entre structures narratives et structures familiales (voir. The Mother/Daughter Plot: Narrative, Psychoanalysis, Feminism, Bloomington et Indianapolis, Indiana University Press, 1989). 


\section{Une épopée hors-la-loi}

Si le roman est traditionnellement défini comme un genre issu de l'épopée ${ }^{4}$, l'utilisation du surnaturel et les références à l'oralité (le nom de "chant" au lieu de chapitre par exemple) dans le roman de Marchessault rappellent cette lointaine origine. De plus, tous les gestes des membres de la famille de Noria se présentent comme les faits merveilleux et extraordinaires des épopées d'autrefois (la présence de la guerre, l'aviation acrobatique, les épreuves à surmonter, etc.). Paul Zumthor définit ainsi l'épopée:

Récit d'action, concentrant en celle-ci ses effets de sens, économe d'ornements annexes, l'épopée met en scène l'agressivité virile au service de quelque grande entreprise. Fondamentalement, elle narre un combat et dégage, parmi ses protagonistes, une figure hors du commun qui, pour ne pas sortir toujours vainqueur de l'épreuve, n'en suscite pas moins l'admiration. ${ }^{5}$

Si elle est présente au cœur du roman de Marchessault, l'épopée ne s'y trouve pourtant pas comme une représentation fidèle des grands combats auxquels l'épopée traditionnelle nous a habitués. La définition de l'êpopée traditionnelle de Zumthor souligne bien qu'il s'agit d'un "récit d'action [...] [qui] met en scène l'agressivité virlle au service de quelque grande entreprise. Daniel Madelénat affirme parallèlement que *la femme, dans l'épopée, est fréquemment "négative": repos et régression coupables qui retardent l'accomplissement hérö̈que " 6 . Suivant cette définition, Marchessault ne peut s'inscrire dans cette généalogie littéraire épopée-roman où la femme écrivaine est totalement absente et de surcroît dépréciée. Pour s'inscrire dans l'histoire littéraire, Marchessault s'approprie l'épopée et elle en subvertit quelquesunes des caractéristiques afin de sortir de l'institution littéraire patriarcale.

4 Dans une étude sur l'épopée à travers l'histoire, Daniel Madelénat affirme qu'entre le roman et l'épopée -les parentés génétiques et structurelles sont claires, du moins en Occident : les premiers romans [...] sont des adaptations comiques, ironiques, sentimentales de l'épopée dont ils se discernent mal parfois [...] - (L'épopée, Paris, Presses Universitaires de France, coll. * Littératures modernes :, 1986, p. 129).

5 Paul Zumthor, Introduction à la poésie orale, Paris, Seuil, coll. *Poétique *, 1983, p. 105.

6 Daniel Madelénat, $o p$. cit., p. 131. 


\section{La maison de la lesbienne}

La narratrice principale, Jeanne, poursuit une quête d'écriture qui sera le leitmotiv du roman de Marchessault: elle désire "sauver le vieux monde" mais aussi s'interroger quant à la famille et surtout quant au père, pivot de ce "vieux monde" patriarcal. Pourtant, lorsque vient le temps de parler du père, Jeanne éprouve la peur du silence. Patricia Smart a montré que les structures narratives sont empreintes de l'idéologie patriarcale (d'où la raison du titre de son essai Écrire dans la maison du përe ${ }^{7}$ ), elle a aussi exploré comment certains textes de femmes subvertissent ces structures et ces "silences" infligés. D'autre part, Jovette Marchessault affirme qu'elle est

de celles dont l'écriture est issue de la longue tradition orale des femmes. [...] J'ai maintenant un langage écrit, poursuit-elle, pour parler de moi-même, de mon rapport au monde. [...] J'affirme que ce langage est politique : qu'il me permet d'émerger, d'agir consciemment, en connaissance de cause, avec la possibilité de l'engagement total de moi-même. ${ }^{8}$

Dans son roman, la présence des voix féminines surnaturelles (les voix de Harriet et de la mère de Noria qui passent par le personnage de l'ange) ainsi que la dénomination de "chant" au lieu de chapitre, comme nous l'avons déjà soulevé, marquent le désir de s'inscrire dans cette "longue tradition orale des femmes", une façon de tracer une origine littéraire féministe. Ainsi, les références à l'oralité propres à l'épopée répondent ici au désir d'inscrire les traces de cette tradition orale des femmes dans le roman Des cailloux blancs pour les forêts obscures. L'écriture de Marchessault rattachée à la tradition orale des femmes est un chant qui outrepasse les contraintes de l'institution littéraire patriarcale. Elle tente en quelque sorte de désaliéner la parole de l'écrivaine et de poursuivre la tradition d'un langage au féminin.

Si on s'en tient aux dires de Jeanne, la rencontre de la fille et du père ne peut advenir que par un "événement surnaturel", presque par un miracle:

7 Patricia Smart, Ecrire dans la maison du père. L'émengence du féminin dans la tradition littéraire du Québec, Montréal, Québec/Amérique, coll. - Littérature d'Amérique *, 1988.

8 Jovete Marchessault, -Ainsi qu'une pluie cyclonale , Le Devoir, 19 avril 1980, p. 25. 
Les Pères? Chaque fois que je me pose cette question, un bref instant, je perds le pouvoir de m'exprimer. [...] Je me sens une pauvre d'esprit en face de cela... Et qui voulant montrer l'approche d'un événement surnaturel, n'a pour l'exprimer qu'un pauvre petit langage à peine créé (p. 13, nous soulignons).

La fille est-elle acculée ici au silence dans la maison du Père? Jeanne ne perd-elle pas le pouvoir de s'exprimer parce qu'elle doit affronter la Loi du Père? Dans le roman de Marchessault, si la rencontre du père et de la fille ne peut advenir que par un "événement surnaturel ", c'est peut-être parce que cette rencontre ne peut exister qu'en dehors de cette maison (celle du Père), dans un lieu "hors-la-Loi".

La création de cet autre lieu, surnaturel, advient grâce à l'écriture de la fille. Cette parole revendique un "nouvel espace familial " - surnaturel et hors-la-loi - : un nouvel espace où le père rencontre la fille, où des voix nouvelles peuvent se faire entendre. Marchessault s'approprie donc l'épopée en détournant entre autres deux de ses caractéristiques traditionnelles: l'oralité est dorénavant rattachée à la tradition orale des femmes et le surnaturel sert à enfreindre les frontières de la maison patriarcale afin de permettre la rencontre de la fille et du père.

Si, pour Patricia Smart, l'espace de l'écriture se situait dans la maison du père, nous découvrons dans le roman de Marchessault que l'écriture se situe dans la maison de la fille lesbienne, la maison de Jeanne et de Noria. Cette maison serait en fait la métaphore d'une organisation différente des rapports entre les êtres, une organisation qui a pris naissance à la suite de l'exclusion d'un de ses membres: la lesbienne. Bâtie selon les désirs d'un personnage lesbien qui a connu l'exclusion et les affres de la Loi du Père (Noria et sa mère étant mortes, Jeanne devient l'unique porte-parole de l'univers lesbien), cette nouvelle maison ne se veut pas à son tour exclusive. Mais tout de même, dans cette maison, chacun et chacune doit fournir un effort: on y entend des voix de femmes qui sont en transformation (la femme du Lion, Noria et Jeanne) et aussi la voix d'un homme qui remet en question son identité patriarcale (le Lion de Bangor). 
$\overline{102}$

\section{Une famille surnaturelle}

Tout comme l'épopée, le roman de Marchessault comporte des événements extraordinaires ou surnaturels et présente un univers où se mêlent le vrai et le merveilleux. Cet univers s'organise de façon vraisemblable sans qu'il y ait d'écart entre les événements étranges et réalistes: la voix de la mère morte que la fille entend dans la radio de son avion, le Lion qui écoute un ange de pierre lui parler, etc.; ces événements arrivent sans étonnement de la part des personnages.

Dans son étude sur le fantastique, Todorov utilise une méthode comparative afin de définir les différences entre le merveilleux, le fantastique et l'étrange?. Un survol d'études plus récentes sur la question de la littérature fantastique montre que les catégorisations apportées par Todorov ne font pas l'unanimité. Dans son étude sur le récit fantastique féminin, Lise Pelletier propose une restitution de "l'ambiguité première du fantastique" 10 . Pelletier soulève d'autre part que "chez. Kathryn Hume, le genre englobant "fantasy" se définit essentiellement par le désir de changer, d'altérer la réalité plutôt que de l'imiter""1. Aussi ajoutet-elle qu'،il est vrai que le refus d'observer certaines règles du réalisme telles que l'unité de temps et d'espace, la distinction entre objets animés et inanimés ou vie et mort, libère ce type de récits de nombreuses contraintes" 12 . L'aspect important de la définition de Hume réside dans le "désir de changer la réalité", c'est du moins ce qui se rapproche le plus du roman de Marchessault qui, par la création d'un nouvel espace hors-la-loi comme nous l'avons vu, désire transformer les structures familiales et les rôles joués par les différents membres de la famille.

Dans le roman de Marchessault, le surnaturel, tout ce qui dépasse la compréhension, advient par des voix surgies de l'audelà que l'on retrouve dans deux passages essentiels du roman:

9 Tzvetan Todorov, Introduction à la littérature fantastique, Paris, Seuil, 1970.

10 Voir Irène Bessière, Le récit fantastique, Paris, Larousse, 1974, p. 12. Lise Pelletier résume ainsi la pensée de Bessière dans Le récit fantastique féminin. (Cabier 39), Québec, Les Cahiers de recherche du GREMF, Université Laval, 1990 , p. 60.

11 Kathryn Hume, Fantasy and Mimesis: Responses to Reality in Western Literature, New York et Londres, Methuen, 1984, p. 21, citée et traduite par Lise Pelletier, op. cit., p. 60.

12 Lise Pelletier, op.cit, p. 61. 
la voix de la mère décédée qui se fait entendre par la radio de l'avion de Noria et les voix de Harriet et de la mère s'exprimant par l'intermédiaire d'un ange de pierre ${ }^{13}$.

\section{Des voix transgressives}

Nous constatons à partir de ces deux passages du roman que le surnaturel apparaît par l'intermédiaire de la voix des femmes, Harriet et la femme du Lion, mais en fait surtout par l'intermédiaire de cette dernière. C'est donc dire que le surnaturel se voit pris en charge par les personnages féminins et notamment par la mère.

Plusieurs théoriciennes ont soulevé le rôle transgressif de la voix féminine, et particulièrement celle de la mère. Selon Luce Irigaray, à l'intérieur des murs de la société patriarcale, "le père interdit le corps-à-corps avec la mère " 14 . En réaction contre cet état de fait, Irigaray ajoute que " ni la petite fille ni la femme n'ont à renoncer à l'amour pour leur mère" 15 . De même, en affirmant que le type de relation qu'entretiennent la mère et la fille se différencie de celle du père et du fils (de l'histoire d'Oedipe), l'étude de Marianne Hirsch sur les voix de la mère et de la fille montre le rôle transgressif que jouent ces personnages dans le développement du roman et dans l'organisation familiale traditionnelle.

Il est intéressant de constater que la fonction du sumaturel tel que le définit Todorov "est de soustraire le texte à l'action de la loi et par là même de la transgresser" 16 . Si la transgression agit dans le déroulement du récit, Todorov ne voit pas dans le surnaturel qu'une fonction littéraire, il lui donne également une fonction sociale:

On voit enfin en quoi la fonction sociale et la fonction littéraire du surnaturel ne font qu'un: il s'agit ici comme là d'une transgression de la loi. Que ce soit à l'intérieur de la vie sociale ou du récit, l'intervention de l'élément surnaturel constitue toujours

13 Précisons qu'aucun nom propre n'est attribué au personnage de la femme du Lion dans le roman. Parfois la nomme-t-on * femme du Lion - et parfois • mère de Noria..

14 Luce Irigaray, Le corps-à-corps avec la mère, Montréal, La Pleine lune, 1981, p. 21.

15 Jbid., p. 31.

16 Tzvetan Todorov, op. cit., p. 167. 
104

une rupture dans le système de règles préétablies et trouve en cela sa justification ${ }^{17}$.

Partant de cette conception de la fonction du surnaturel, nous pouvons voir que les voix féminines en tant que phénomènes surnaturels jouent donc un rôle de transgression de la Loi patriarcale, autant à l'intérieur de la trame narrative que de l'organisation familiale. Si l'histoire de la famille de Noria semble atteindre un cul-de-sac après la mort de Harriet et de la mère de Noria, l'exploitation du surnaturel dans la narration permet de pousser plus loin les relations qu'entretiennent les personnages. C'est par la voix de l'ange dans le cimetière que le père peut reprendre contact avec sa femme et Harriet. Et c'est la voix de la mère de Noria, alors décédée, qui se fait entendre par la radio pour sauver Noria d'un accident d'avion et la mener vers Jeanne. La voix féminine cause une rupture dans la linéarité du récit mais aussi dans la linéarité de la généalogie familiale. Ces phénomènes permettent de sortir du carcan de la famille traditionnelle, laissant place à de nouvelles relations entre les êtres.

\section{Une brèche dans le ciel : la voix de la mère morte}

Le passage où l'on retrouve la voix de la mère dans la radio de bord de la fille est particulièrement déterminant. Si la mère de Noria était déjà décédée depuis plusieurs années lorsque Jeanne et Noria se sont rencontrées, la mère a tout de même joué un rôle dans leur rencontre. Noria raconte à Jeanne cette histoire de vol où elle fut prise dans une tempête en Nouvelle-Angleterre. Presque certaine d'y laisser sa vie, elle a survécu: c'est la voix de sa mère, morte depuis longtemps, qui l'a guidée et sauvée. Dans une solitude essentielle devant l'imminence de la mort alors que son avion perd de l'altitude, Noria entend comme par miracle la voix de la mère :

Soudain, [ . . ] quelqu'un était en train d'agrandir les ondes sonores et d'élargir nos tympans. [... L La radio de bord me communiquait une succession d'impressions [...] quand j'ai entendu la voix de ma mère morte il y a longtemps, si longtemps... - Vers les cimes! Vers les cimes, hurla encore plus fort la voix de ma mère (p. 77) 
Dans ce passage, on note que la voix de la mère élève, grandit ("agrandir les ondes", "élargir les tympans"). Elle pousse sa fille vers l'audace salvatrice ("vers les cimes") et l'incite à passer par une brèche. Par-delà la mort, la mère crée 'une ouverture dans les nuages, dans la montagne, mais aussi dans la trame du récit traditionnel:

- Mère, je n'en reviendrai pas!

- Il y a une brèche! Tu vas passer.

Là, devant moi, la muraille est fendue en deux [...] (p. 78).

À ce moment, pour leur première rencontre, c'est vers Jeanne que Noria se dirigeait, guidée par sa mère. Si la mère de Noria meurt, elle ne disparaît pas du récit. Au contraire, elle incarne la force qui ouvre, qui fait éclater (la "brèche") le récit et la famille. La voix de la mère outrepasse les frontières entre la vie et la mort pour sauver sa fille et la guider vers Jeanne. Si cette situation laisse voir l'affiliation entre la mère et la fille, elle permet également d'observer l'attachement entre une mère lesbienne et sa fille lesbienne. Aussi, en provoquant une rencontre entre sa fille Noria et Jeanne, la mère crée une brèche non seulement dans le récit traditionnel, mais aussi dans le monde patriarcal. En tant que lesbienne, le personnage de la mère s'écarte déjà du rôle traditionnel de la mère de famille. Si la mère n'a pu se sauver elle-même dans le monde du Lion, elle a sauvé sa fille. Le surnaturel permet une continuité, une solidarité des générations féminines. Dans le développement du récit, l'utilisation de la voix de la mère morte permet de mettre en scène une continuité dans les rapports mère-fille qui prend toute son importance si on se réfère au travail de Luce Irigaray (le corps-à-corps avec la mère) et de Marianne Hirsch (qui parle de faire entendre la voix de la mère). Cette solidarité entre la mère et la fille se retrouve dans d'autres aspects du roman puisque Noria est comme sa mère pour l'essentiel: le métier d'aviatrice, son orientation sexuelle, les valeurs humanitaires de sa jeunesse. De ce point de vue, la mère survit dans sa fille, tout comme la fille survit grâce à la voix de la mère morte.

Cette apparition surnaturelle permet également de faire en sorte que le personnage de la mère continue à être un facteur qui bouleverse la vie du Lion: par elle, Noria quittera le.Lion pour Jeanne, ce qui met le père face à une seconde relation lesbienne (sa femme avec Harriet ayant été la première). La venue du 
106

second couple lesbien (Jeanne et Noria) et les circonstances entourant son dénouement (la mort de Noria) permettent une rencontre entre l'amante lesbienne et le père. L'événement surnaturel de la voix de la mère dans la radio de l'avion a donc des répercussions énormes dans le déroulement du récit ainsi que dans la reconstruction de la famille de Noria. Ainsi, le surnaturel est un élément structurant essentiel. Comme si, pour réinventer la famille - et le roman -, il ne fallait pas moins qu'un miracle pour que s'ouvre une brèche dans la famille et dans la textualité.

\section{Les héroïnes et les héros changent de peau}

Par l'affirmation d'une tradition féminine de l'écriture et l'utilisation de voix féminines transgressives, Marchessault réinvente une forme nouvelle de l'épopée à l'intérieur de son roman. Contrairement à l'«agressivité virile " de l'épopée traditionnelle, l'épopée est là "pour nous rapprocher". C'est ainsi que Jeanne clôt le roman sur ces mots: "Le petit avion est toujours au bord de l'êtang. Il est beau comme une épopée qui serait passée dans nos âmes, pour nous rapprocher. Beau, comme une pensée de confiance" (p. 165).

Nulle trace de combat contre l'autre, aucune recherche d'admiration. S'il y a combat, c'est à l'intérieur de chaque personnage, avec lui-même. La "grande entreprise" de l'épopée consiste ici à réinventer la famille, mais, loin de servir cette cause, l'^agressivité virile" y nuit. Il faut déposer les armes et chercher la vérité en soi. Les personnages mis en scène ne sont pas une idéalisation des parents mais des héros différents qui se donnent l'occasion de réévaluer leur rôle.

Dans sa jeunesse, le Lion se présente sous l'image du héros traditionnel de l'épopée, un homme hors du commun et admiré de tous. Avide de réussite et défiant toute compétition, il accorde à sa femme dès les premiers temps de leur mariage le rôle d'une rivale: "La femme que j'aimais était de taille à donner naissance à une légende et tel un acide, la jalousie et l'envie passaient sur moi " (p. 133). Lorsque sa femme le quitte pour Harriet, la rivale du début devient aussitôt une ennemie à éliminer. Dévoilant son plan, le Lion se compare justement au héros de l'épopée:

L'excitation de la vengeance revenait, inavouable. Dans une épopée, $[\ldots]$ on mesure la force, le rayonnement du héros à la 
taille des épreuves qu'il doit surmonter. [...] Si, pour le commun des mortels, la vengeance est un plat qui se mange froid, pour le véritable héros, c'est un devoir: le devoir de riposter (p. 144).

Le Lion ne se considère pas ici comme faisant partie du commun des mortels; il est un héros pour qui la vengeance est un devoir. Il lui faut dès lors combattre. Ce combat contre l'autre est au cœur de l'épopée traditionnelle telle que la définit Zumthor: " le chant épique narre le combat contre l'Autre, l'étranger hostile, l'ennemi extérieur au groupe - que ce dernier soit une nation, une classe sociale ou une famille. 18 .

Pour le Lion, l'ennemi, c'est sa propre femme et Harriet. De son côté, la femme du Lion semble se situer dans une "autre" réalité. Aussi lui rappelle-t-elle qu'il n'est pas un héros d'épopée, mais un simple être humain:

Je vais partir avec ma fille. Nous avons le droit d'échapper aux égouts où ton existence s'est égarée. En matière de vengeance, tu es une sorte de maître. [...] L'être humain n'a pas droit à la vengeance! (p. 147)

La réplique de la femme du Lion conteste la définition que le mari se fait d'un héros et affirme une vision autre de l'existence. En quittant la maison du mari, elle signifie qu'elle s'oppose à une structure narrative traditionnelle où l'homme joue le rôle du héros guerrier et la femme celui du protagoniste négatif qui "retarde l'accomplissement héroïque .

La vie des personnages de la famille de Noria sont à la hauteur d'une épopée, mais il y a remise en question des valeurs traditionnelles inhérentes à ce genre par la transformation du rôle du héros. C'est une héroïne qui affirme que «l'être humain n'a pas droit à la vengeance"; par la suite, le héros, le Lion, remettra son rôle en question. La nature de la quête du héros est donc subvertie par une critique venant de la mère de Noria.

Le féminisme, par sa quête de nouvelles relations entre les sexes, conduit à revoir les rôles masculins et féminins. Marchessault "féminise" donc l'épopée non seulement en incluant des personnages féminins avec de nouveaux rôles (la mère lesbienne) mais également en transformant les rôles traditionnels joués autant par les hommes que par les femmes. Il ne s'agit pas 
108

ici d'une inversion des rôles masculins et féminins mais de l'affirmation de rôles individuels surpassant les rôles sociaux préétablis ${ }^{19}$.

Après le départ de sa femme, il faut se rappeler le déroulement de l'histoire du Lion et tout le processus de réflexion dans lequel il s'engagera. Ce rôle de héros au cœur duquel il s'enfermait deviendra étouffant et il remettra en question ce monde du passé, celui appartenant à l'épopée et au patriarcat. Le père vengeur de l'épopée s'humanise peu à peu. Jovette Marchessault ne met pas en scène "une "figure hors du commun tel le héros traditionnel défini par Zumthor. D'ailleurs, dans ce roman, il n'y pas "une" figure exceptionnelle mais une série de personnages en quête qui prennent la parole - et la vedette - à tour de rôle : le Lion, sa femme, Harriet, Noria et Jeanne. De manière plus globale, le roman remet en cause la notion du héros solitaire. Parlant de la venue d'une nouvelle définition du rôle de héros dans les textes féministes, Jean Pfaelzer soulève justement que: "With multiple, fragmented, imperfect, and ordinary heroines, these texts subvert the patriarchal assumption of the hero as solitary, individualistic, isolated, contentious, and victorious" 20 .

La présence d'héroïnes multiples féminise un genre traditionnellement masculin. Cette révision du héros vise à s'opposer aux genres traditionnels où les rôles des protagonistes sont totalement figés et stéréotypés, et cela, autant pour le modèle masculin (le rôle actif) que féminin (le rôle passif). Dans son étude sur le roman, Anne Cranny-Francis constate effectivement que les rôles attribués aux hommes et aux femmes sont extrêmement conservateurs et soulève que les écrivaines féministes doivent développer des stratégies innovatrices pour contourner ce qui apparait d'emblée "a no-win situation" 21 .

L'épopée dans le roman de Marchessault est donc subvertie par le fait qu'elle ne mèt pas "en scène l'agressivité virile" mais s'évertue plutôt à déconstruire cette agressivité tout en transfor-

19 Voir à ce sujet Dana A. Heller, The Feminization of Quest-Romance: Radical Departures, Austin, Texas Press, 1990, p. 122.

20 Jean Pfaelzer, * Response. What Happened to.History?•, Libby Falk Jones et Sarah Webster Goodwin (dir.), Feminism, Utopia, and Narratice, Knoxville, University of Tennessee Press, 1990, p. 197.

21 Anne Cranny-Francis, Feminist Fiction: Feminist Uses of Generic Fiction, Cambridge (Angleterre), Polity Press, 1990, p. 19. 
mant la notion de héros: la mère quitte la maison du père en refusant ses valeurs de violence et le Lion, isolé, finit par rejeter son identité patriarcale. Marchessault intègre dans son roman des attributs de l'épopée - un genre traditionnellement masculin, viril et les transforme en les rapprochant de préoccupations et de stratégies narratives féministes. L'utilisation explicite de l'épopée familiale dans sọn roman lui sert à se positionner face à la tradition et à questionner les normes fixées par l'institution littéraire. Ainsi, elle révise un genre littéraire traditionnel (féminisation de l'épopée dite "virile") et transforme la structure familiale patriarcale (père, mère et fille: des héros qui n'en sont plus). Vis-à-vis de l'histoire littéraire, Marchessault affirme une appartenance autre, lui venant de la "longue tradition orale des femmes", mais cela ne la conduit pas pour autant à rejeter en bloc la tradition littéraire.

\section{Un roman métaféministe}

Le roman de Marchessault fait référence à l'épopée non pas pour la renier mais pour la réinvestir de nouvelles valeurs: l'épopée n'est pas détruite mais réinventée. C'est tout comme la famille. L'épopée familiale, cette grande fresque de vie, n'est plus combat et défense mais rapprochement et "pensée de confiance". De cette façon, le roman aspire à un futur qui ne sera pas coupé du passé. Par son attachement à la tradition orale des femmes et par son désir d'ouvrir les frontières du genre traditionnel et de la famille, le roman de Marchessault s'inscrit dans une pensée "métaféministe (un terme proposé par Lori Saint-Martin en opposition à l'appellation "postféministe"), une pensée de continuité et de solidarité :

Pas plus que le métalangage ne tue le langage, le métaféminisme n'annonce le déclin du féminisme; plutôt, il l'accompagne et l'enveloppe [...] Ainsi, les écrits métaféministes affirment autant leur enracinement que leur différence, suggèrent à la fois qu'ils vont plus loin et qu'ils accompagnent, éçoutent, tendent la main. ${ }^{22}$

Le métaféminisme, par l'affirmation de son "enracinement " et de sa "différence", répond en écho à l'épopée de Zumthor qui

22 Lori Saint-Martin, - Le métaféminisme et la nouvelle prose féminine au Québec., Lori Saint-Martin (dir.), L'autre lecture. La critique au féminin et les textes québécois, tome II, Montréal, XYZ, coll. -Documents •, 1994, p. 165. 
"au long de la tradition, [peutl se modifier avec les circonstances". Développant sur la nature même de l'épopée, Zumthor ajoute:

Il n'y a pas d'^âge héroïque *, et le "temps des mythes * n'est pas celui de l'épopée: il n'y a que l'incessante fluidité du vécu, une intégration naturelle du passé au présent. [...] L'épopée n'a rien d'un musée. Il n'y a pas d'histoire à proprement \parler, mais une vérité perpétuellement recréée par le chant. ${ }^{23}$

Du changement inhérent à l'épopée, de la métamorphose propre au récit métaféministe, le roman Des cailloux blancs pour les forêts obscures porte en lui un projet de transformation à l'image de la quête d'écriture de Jeanne: la transformation du roman traditionnel de la famille.

\section{Du surnaturel à l'utopie : un projet politique}

Jovette Marchessault confiait en entrevue que Des cailloux blancs pour les forêts obscures "est un roman prospectif écrit en fin de millénaire, avec de l'espoir pour un futur* et qu'avec ce roman, elle a "essayé d'imaginer comment seront un jour les relations entre les hommes et les femmes, entre les parents et les enfants „24. Par ce projet d' espoir pour un futur", par cette tentative d'imaginer les relations dans les familles de demain, Marchessault inscrit d'emblée son livre dans la lignée du roman utopique. À ce propos, notons que "le féminisme, par sa quête de nouvelles façons de vivre eu égard à l'État et par sa tentative de changer la "nature humaine" telle que les hommes l'ont traditionnellement définie, constitue l'utopie par excellence. ${ }^{25}$.

Si l'utilisation du surnaturel à l'intérieur de l'épopée familiale débouche sur la réconciliation des hommes et des femmes et permet la construction de nouvelles identités, délivrées des rôles traditionnels figés, le roman de Marchessault ne relève pas de l'acception courante de l'utopie, qu'on rattache à la chimère, mais

23 Paul Zumthor, op. cit., p. 109, nous soulignons.

24 Jean Royer, : Le roman de la réconciliation - Le dewoir, 8 octobre 1987, p. 15.

25 Elaine Hoffman Baruch, - The Quest and the Questions *, R. Rohrlich et E.H. Baruch (dir.), Women in Searcb of Utopia, New York, Shocken Books, 1984, p. xi-xii. Guy Bouchard résume ainsi la pensée de Elaine $\mathrm{H}$. Baruch dans - Le rôle des images du futur dans la pensée féministe., Guy Bouchard (dir.), Images féministes du futur, Les Cahiers du Grad, n 6, Québec, Faculté de philosophie, Université Laval, 1992, p. 1. 
bien d'un projet politique concret. Précisons que le roman de Marchessault ne se présente pas comme un roman utopique traditionnel où l'on retrouve habituellement la projection d'une vision sociale dans un monde futur; il n'en contient pas moins un projet utopique par la quête qu'il poursuit. $\grave{A}$ propos de l'utopie, Frances Bartkowski soulève que:

The utopian aspects of feminist theory have always been apparent, from Mary Wollstonescraft to Hélène Cixous. [...] Feminist fictions are the aplaces . where women speak the desires that frame the anticipatory consciousness of utopia made concrete, bringing the not-yet into the here and now. ${ }^{26}$

Le "roman prospectif" de Marchessault donne en effet place au "not-yet" dans le "here and now". D'autre part, Louis Marin soutient que "l'utopie est une critique de l'idéologie dominante dans la mesure où elle est une reconstruction de la société présente (contemporaine) par un déplacement et une projection de ses structures dans un discours de fiction ${ }^{27}$. Démontrant bien la nature politique de l'utopie, Marin affirme qu'elle "est une critique idéologique de l'idéologie ${ }^{28}$. Ce dernier point rejoint la pensée de Bartkowski qui soulève que les romans féministes utopiques sont le lieu où les théories du pouvoir peuvent être critiquées à l'intérieur même des constructions narratives où elles prennent place 29 .

Roman féministe et utopique, le roman Des cailloux blancs pour les forêts obscures de Jovette Marchessault constitue en soi un projet politique qui remet en question les lieux de pouvoir à l'intérieur des structures narratives et des structures familiales. En somme, l'utilisation dans le roman de Marchessault de caractéristiques propres à différentes références génériques, en passant par l'épopée, le roman familial, le surnaturel, l'utopique ainsi que l'autobiographique (dont nous n'avons pu traiter ici), amène une transgression simultanée de la loi du genre littéraire traditionnel, de la loi de la linéarité du récit et de la loi de la famille patriarcale.

26 Frances Bartkowski, Feminist Utopias, Lincoln et Londres, University of Nebraska Press, 1989, p. 162.

27 Louis Marin, Utopiques. Jeux d'espaces, Paris, Minuit, coll. .Critique., 1973, p. 249.

28 Ibid.

29 Frances Bartkowski, op. cit, p. 5. 